\title{
DEVELOPMENT OF NORMAL BINOCULAR VISION IN EARLY CONVERGENT STRABISMUS AFTER ORTHOPHORIA*
}

\author{
BY \\ GIOVANNI MARAINI AND LUIGI PASINO \\ From the University Eye Clinic, Parma, Italy
}

A fundamental difference exists, from the sensory viewpoint, between the cases of constant squint developing before the third year of life and all the other forms of oculomotor imbalance. The first group is the one considered in the literature as that of "strabismus with anomalous correspondence".

Must we consider the "anomalous correspondence" of these subjects as a system of corresponding retinal points similar in its organization to normal correspondence? A great divergence of opinion and of statistical evidence with regard to this problem is found in the literature; we think that these discrepancies may be easily explained by the fact that different and unnatural tests were used to assess the state of retinal correspondence.

The systematic examination of squinting subjects in normal surroundings (tests with striated glasses) allows certain conclusions to be drawn for subjects under 10-12 years of age:

(1) The occurrence of a definite post-operative diplopia or a relapse of the original angle of deviation is exceptionally rare in these subjects; this would not be so if anomalous correspondence was a rigid system comparable to that of normal correspondence. Moreover, anomalous retinal correspondence only rarely forms the sensory basis for the compensation of a small oculomotor imbalance (rare fusional movements) (Maraini and Pasino, 1964).

(2) Anomalous retinal correspondence adapts itself easily and rapidly to the changed angle of deviation brought about by surgical treatment. It would seem that, in the absence of amblyopia or of deep suppression, the sensory behaviour in normal surroundings depends chiefly on the position of the eyes (Pasino and Maraini, 1964). As this is changed by surgery to very small angles (without vertical deviation) the anomalous binocular vision of these subnormal conditions is characterized by harmonious anomalous retinal correspondence with fusion, a fusional amplitude nearly exclusively based on variations of the anomaly and only rarely on fusional movements (Maraini and Pasino, 1964), and a coarse stereopsis with very low stereoscopic acuity (Pasino, Maraini, and Santori, 1963). If orthophoria is obtained (with prisms, for instance) one would expect this sensory situation to become converted into a normal binocular localization (Bagolini, 1961).

In the last few years we have had the opportunity of treating and following up for a sufficient interval of time a certain number of these patients. All of them had a fixed convergent strabismus of very early onset with anomalous retinal correspondence unresponsive to orthoptic treatment. After perfect orthophoria for far 
and near vision had been obtained (by surgery and nearly always by additional prismatic glasses) the possible development of normal binocular vision could be adequately estimated by frequent examination. The sensory state was always examined in normal surroundings with Bagolini's striated glasses. The presence of fusional movements or of variations of the retinal correspondence in free space was investigated by a technique previously described (Maraini and Pasino, 1964). Stereoscopic acuity was tested by a modification of the two-rods test (Pasino and others, 1963).

\section{Case Reports}

\section{Some illustrative cases are briefly described.}

Case 1, aged 10 years. Surgery at the age of $3 \frac{1}{2}$ years. The deviation was reduced to $+8^{\Delta}$ with harmonious anomalous correspondence in free space. Orthophoria was obtained by optical correction after four years of unsuccessful orthoptics. After more than one year the patient is still orthophoric with normal binocular localization in normal surroundings; fusional movements are still absent and the fusional amplitude is exclusively based on variations of the retinal correspondence. Stereoscopic acuity does not reach low normal values.

Case 2, aged 10 years. No treatment up to the age of 7. A first surgical operation reduced the deviation to $+8^{\Delta}$; retinal correspondence was anomalous at the synoptophore and harmoniously anomalous in free space. A second operation reduced the angle to $+4^{\Delta}$ but the sensory state did not change. Orthophoria was obtained by prisms and normal binocular localization was immediately present in normal surroundings. Without further treatment retinal correspondence was definitely normal at the synoptophore after 10 months. After $1 \frac{1}{2}$ years fusional movements are present with base-out prisms, while variations of retinal correspondence are elicited with base-in prisms. Stereoscopic acuity is in the low normal range.

Case 3, aged $7 \frac{1}{2}$ years. No treatment up to the age of 7 when orthophoria was obtained by surgery and prisms. After orthophoria was gained retinal correspondence was still clearly anomalous at the synoptophore. Without orthoptic treatment, after 4 months the patient is orthophoric with convergent fusional movements up to $15^{\Delta}$. Stereoscopic acuity absent.

Case 4, aged 8 years. Orthophoria obtained by surgery at the age of 4 . Some orthoptic treatment was given but the fusional amplitude did not exceed $5^{\circ}$ although retinal correspondence was normal. After $3 \frac{1}{2}$ years the patient is still orthophoric with good fusional vergences but stereoscopic acuity is lacking.

Case 5, aged 14 years. No treatment up to 9 years when surgery reduced the deviation to a small convergent angle with harmonious anomalous retinal correspondence in normal surroundings. Normal retinal correspondence was obtained after orthoptic treatment, but the sensory condition in free space was unchanged. Orthophoria was reached by the aid of prisms with normal binocular localization in normal surroundings. After two years the patient has good fusional movements, diplopia without the prismatic correction, and stereoscopic acuity in the low normal range.

Case 6, aged 7 years. No treatment up to 6 years, when orthoptics failed to transform anomalous retinal correspondence; orthophoria by surgery with immediate normal binocular localization in normal surroundings, while retinal correspondence was still anomalous at the synoptophore. After 2 months, while retinal correspondence is unchanged at the synoptophore, the patient is still orthophoric with convergent fusional movements of $10^{\Delta}$. Stereoscopic acuity is lacking.

Case 7, aged 7 years. After intensive and unsuccessful orthoptics, orthophoria was obtained at the age of 6 by prismatic and bifocal lenses. Normal binocular localization was immediately present in free space while retinal correspondence persisted as anomalous at the synoptophore. After 4 months the patient gave equivocal answers on examination with the synoptophore. After 6 months, orthophoria with normal binocular localization in normal surroundings but, with baseout prisms, fusional movements are frequently substituted by a variation of retinal correspondence; stereoscopic acuity still absent.

Case 8, aged 7 years. After unsuccessful orthoptics, orthophoria was obtained at the age of 7 by surgery and prisms. Normal binocular localization was immediately present in free space. 
(2) This normal binocular localization does not immediately possess the characteristics of a stable functional relationship peculiar to fully developed normal retinal correspondence; on the contrary, it retains all the characteristics of harmonious retinal correspondence on a small-angle strabismus. A fusional amplitude in normal surroundings is generally obtained by means of variations of retinal correspondence and not by fusional movements. Stereoscopic acuity is absent.

(3) If orthophoria is maintained over a sufficient lapse of time and if the patient's binocular vision is still in the plastic stage, this empirical "normal" binocular relationship progressively reaches the characteristics of normal binocular vision. The variations of retinal correspondence following the introduction of a horizontal disparity are slowly replaced by real fusional movements. Finally, stereoscopic acuity appears.

\section{Discussion}

The observation of the gradual development of normal binocular vision in early convergent squint by simple orthophoria raises a question. Must we consider normal retinal correspondence as innate or as empirically acquired?

Two main interpretations are found (Helmholtz, 1856; Hering, 1879). The empirical theory of Helmholtz maintains that the local sign of the retinal elements and the functional relationship between the two retinae are the result of experience. The opposite view is held by Hering's "nativism", by admitting the innateness of the retinal local sign and of retinal correspondence. Both these theories are based on indisputable observations: for example, for "empirism" the occurrence of anomalous correspondence in squinting subjects; for "nativism" the existence of discrepancies between visual and objective directions (Tschermak-Seysenegg, 1952). Most modern authors have accepted Hering's theory of nativism.

We think that, as suggested by Kretzschmar (1955), many of the facts considered as the basis for these theories may be grouped together into a single interpretation. The symmetry of the retinal mosaic in the two eyes, and consequently the retinal local sign, is not linked to binocular vision, as it exists even in animals without a binocular visual field. The "nativism" may be applied to the relationship between the different retinal local signs within a single uniocular visual field; this stable organization explains the phenomenon of the discrepancies and seems to be linked to the anatomic organization of the retina and of the visual cortex.

A different situation exists if the binocular visual act is considered, i.e., the relationship connecting the two uniocular visual fields. Following Kretzschmar's (1955) theory, "empirism" could be accepted at this binocular level. In effect, the system of normal binocular correspondence is not so severely rigid and fixed as it would be expected to be if we were dealing with an inborn anatomic organization. This is demonstrated by the fact that, within certain limits, transversely disparate images may be fused (Panum's region of binocular single vision, fixation disparity). A further demonstration of an empirical theory so interpreted is the occurrence of anomalous retinal correspondence as an adaptation to a motor disturbance occurring in early life.

What is then the empirical factor (or factors) leading to the appearance of a functional correspondence between the uniocular visual fields? At the present state of our knowledge a sure answer to this question is difficult, if not impossible. There 
is no question that fusion and stereoscopic vision may occur in lower animals with immobile and/or independent eyes and total optical decussation. On the other hand, it is certain that if a high degree of binocular vision is to be attained an exact retinal correspondence is needed, and this strictly depends on the possibility of extremely exact co-ordinate eye movements ensuring the preservation of a mutual relationship of the two eyes in all gaze directions. This will be obtained (DukeElder, 1958) only if the two eyes are controlled by the cortical centres as a unitary organ. In effect, in phylogenesis, together with co-ordinate eye movements, a partial decussation of the optic pathways appears, with resulting unilateral cortical representation of all the functionally right or left retinal points-an essential condition if co-ordinate eye movements are to occur. On this basis the importance of the motor factor in the genesis of retinal correspondence and of binocular vision seems therefore clear, and the experience of the first years of life appears to be of paramount importance. In our opinion, the value of the empirical theory is limited to this particular, but extremely important, period. It would seem therefore logical to assume that retinal correspondence does not develop if an oculomotor imbalance appears during this period. This seems to be the case for constant manifest strabismus of early onset (before the third birthday); the harmonious anomalous retinal correspondence of these subjects seems to represent a labile sensory adaptation to the ocular deviation which immediately converts into a normal binocular localization as the angle of deviation disappears. In our opinion this behaviour would not seem to be linked to the reappearance of a pre-existing normal retinal correspondence, but to the settling of an empirical functional relationship between the two retinae. Because of the absence of the deviation this normal binocular localization continuously approaches normal correspondence without reaching, for a long time at least, the characteristics of a stable organization which are peculiar to normal retinal correspondence and to normal binocular vision.

\section{Summary}

The possibility is discussed, and some evidence is presented, that the development of normal binocular vision in early convergent strabismus after orthophoria has been obtained, is not linked to the reappearance of a pre-existing normal binocular correspondence, but to the gradual development of an empirical functional relationship between the two retinae. Binocular normal localization is immediately gained with the orthophoria, but normal fusional movements and, finally, normal stereoscopic acuity appear at a much later date.

\section{REFERENCES}

Bagolini, B. (1961). · Ann. Oculist. (Paris), 194, 236.

DuKE-Elder, S. (1958). "System of Ophthalmology", vol. 1, p. 698. Kimpton, London.

HeLmHOLTZ, H. von (1856). "Handbuch der physiologischen Optik". Voss, Leipzig.

HeRING, E. (1879). " "Der Raumsinn und die Bewegungen des Auges". In "Handbuch der Physiologie" ed. L. Hermann, band 3, teil 1, pp. 343-601. Vogel, Leipzig.

Kretzschmar, S. (1955): Docum. Ophthal., 9, 46.

Maraini, G., and Pasino, L. (1964). Brit. J. Ophthal., 48, 439.

Pasino, L., and Maraini, G. (1964). Ibid., 48, 30.

- - and SANTORI, M. (1963). Ann. Ottal., 89, 1005.

Tschermak-SeysenegG, A. von (1952). "Introduction to Physiological Optics", trans. P. Boeder, p. 135. Thomas, Springfield, IIl. 